\title{
Effects of late-gestation heat stress on immunity and performance of calves ${ }^{1}$
}

\author{
G. E. Dahl, ${ }^{* 2}$ S. Tao, $†$ and A. P. A. Monteiro† \\ *Department of Animal Sciences, University of Florida, Gainesville 32611 \\ †Department of Animal and Dairy Science, University of Georgia, Tifton 31793
}

\section{ABSTRACT}

Lactating cows that experience heat stress will have reduced dry matter intake and milk yield and shift metabolism, which ultimately reduces the efficiency of milk production. Dry cows that are heat stressed similarly experience lower intake, reduced mammary growth, and compromised immune function that ultimately results in a poorer transition into lactation and lower milk yield in the next lactation. A recent focus in our laboratory is on the effects of late gestation, in utero heat stress on calf survival and performance. We have completed a series of studies to examine preweaning growth and health, and later reproductive and productive responses, in an attempt to quantify acute and persistent effects of in utero heat strain. Late gestation heat stress results in calves with lower body weight at birth, shorter stature at weaning, and failure to achieve the same weight or height at 12 mo of age observed in calves from dams that are cooled when dry. A portion of the reduced growth may result from the lower immune status observed in calves heat stressed in utero, which begins with poorer apparent efficiency of immunoglobulin absorption and extends to lower survival rates through puberty. Heat-stressed calves, however, have permanent shifts in metabolism that are consistent with greater peripheral accumulation of energy and less lean growth relative to those from cooled dams. Comparing reproductive performance in calves heat stressed versus those cooled in utero, we observe that the cooled heifers require fewer services to attain pregnancy and become pregnant at an earlier age. Tracking the milk production in calves that were heat stressed in utero versus those cooled in late gestation revealed a significant reduction of yield in the first lactation, approximately $5 \mathrm{~kg} / \mathrm{d}$ through $35 \mathrm{wk}$ of lac-

\footnotetext{
Received June 19, 2015.

Accepted September 28, 2015.

${ }^{1}$ Presented as part of the Animal Health: Understanding and Reducing the Impact of Various Stressors on Immune Responses and Health of Cattle Symposium at the ADSA-ASAS Joint Annual Meeting, Orlando, Florida, July 2015.

${ }^{2}$ Corresponding author: gdahl@ufl.edu
}

tation, despite similar body weight and condition score at calving. These observations indicate that a relatively brief period of heat stress in late gestation dramatically alters the health, growth, and ultimate performance of dairy calves. Thus, it is critical to effectively manage heat stress of dry cows to avoid negative effects on the calf.

Key words: calf, in utero heat stress, growth, health

\section{INTRODUCTION}

Heat stress causes significant negative responses during all phases of the life and production cycle of dairy cattle. Indeed, when lactating cows experience heat stress, they reduce intake, alter metabolic priorities, and eventually suffer a reduction in milk yield (Collier et al., 2006; Baumgard and Rhoads, 2013). Calves also suffer under heat stress, with dramatic reductions in growth apparent before and after weaning (Broucek et al., 2009). Of interest, Broucek et al. (2009) observed lower growth without reductions in feed intake, suggesting a reduction in feed efficiency of heat-stressed calves relative to those under moderate environmental conditions. During the postweaning growth phase, heat stress reduces ADG of calves (O'Brien et al., 2010). In contrast to mature cows, this growth decrement can be explained by reduced DMI alone rather than the interaction of lower DMI and metabolic shifts (Rhoads et al., 2010). It is clear, however, that heat stress reduces productivity of growing and lactating cows.

In addition to the negative effect on production, heat stress may also depress immune function in cows, although evidence of direct effects is scarce. Elvinger et al. (1992) reported a reduction in circulating leukocyte migration into the mammary gland under heat stress relative to thermoneutral conditions, and the effect was independent of yield as bST treatment did not alter the response to heat stress despite greater milk production. Indirect evidence of negative effects of heat stress on immune status in cows is apparent from examination of seasonal effects on peripheral blood mononuclear cell (PBMC) proliferation, where DNA synthesis was consistently decreased in cows calving in the summer relative to spring (Lacetera et al., 2005); however, IgM 
secretion from those same PBMC was decreased in spring compared with summer, suggesting an inconsistency of immune response to heat stress. Relative to thermoneutrality, calves under chronic heat stress have decreased IgG production and increased mortality (Kelley et al., 1982), further support for the concept that hyperthermia reduces immune status in old and young cattle. But less is known regarding the effect of in utero heat stress on calf health and performance.

Beyond the negative outcomes in lactating and growing dairy cattle, heat stress during the dry period significantly affects the success of the transition and reduces production efficiency in the next lactation (Tao and Dahl, 2013). Specifically, heat-stressed dry cows have reduced mammary growth, poorer immune status in the transition, and lower subsequent milk yield relative to cows that are cooled for the entire dry period. Further, previous studies and our recent work suggest that the negative effects of heat stress during late pregnancy result in substantial adverse effects on the developing calf before and after birth. Ongoing studies in our laboratory have revealed a series of shifts in immune, metabolic, and performance outcomes that result from a brief period of heat stress in late gestation. These changes in metabolism and health appear to have lifelong effects on the calf, and reduce the productive potential of that calf later in life.

\section{HEAT STRESS EFFECTS ON CALF DEVELOPMENT}

It is important to begin with a description of how we manage cows during the dry period to gain perspective on the relatively brief duration and limited level of heat stress that can result in substantial reductions in performance. In all of our controlled studies, multiparous cows are used; that is, they have calved at least once previously and are drying off after a typical lactation. The treatment we impose is simple; cows are milked for a final time, dry cow antibiotic therapy is given along with teat sealant, and the cows shift to a lower energy diet than they were consuming during lactation. Cows are housed in a typical freestall barn with sand beds, and the treatment consists of turning the fans and feedline soakers off in half of the barn, which results in half of the cows having only shade (i.e., heat stressed) relative to the other cows that are actively cooled with fans and soakers under shade (i.e., cooled). Relative to cooled cows, heat-stressed cows typically show a consistent 0.3 to $0.4^{\circ} \mathrm{C}$ rise in average rectal temperature and an 1.5- to 2-fold increase in respiration rate. These responses appear within a day of treatment and persist for the entire dry period. Thus, the calves developing in the uteri of heat-stressed dams are exposed to increased maternal core temperature and respiration rate during the final 6 to 7 wk of gestation. Despite the relatively modest level and duration of heat stress, significant negative outcomes result in the calf.

In confirmation of earlier work with cows maintained on pasture with shade or without during the dry period (Collier et al., 1982), actively cooling dry cows had profound positive effects on calf birth weight relative to those that were heat stressed (Tao et al., 2012). Thus, even under improved housing conditions in a free-stall environment, in utero heat stress results in a smaller calf at birth relative to cows that are cooled. Of interest, that birth weight difference was maintained at weaning despite the fact that weight gain after birth did not differ between calves heat stressed in utero and those born to cooled dams (Tao et al., 2012). Gestation length is also reduced with heat stress during the dry period, typically by 4 to $5 \mathrm{~d}$, which accounts for a portion of the smaller calf size (Tao and Dahl, 2013). Lighter birth weight likely results primarily from placental insufficiency during late gestation, as heat stress decreases bovine placental weight (Collier et al., 1982) and function, as indicated by reduced placental hormone production including estrone sulfate, placental lactogen, and pregnancy-associated glycoprotein (Collier et al., 1982; Bell et al., 1989; Thompson et al., 2013). But lower maternal intake is also a factor in the lower birth weight of heat-stressed calves. Further evidence that metabolic adaptation to heat stress differs in the dry period versus lactation is found in a recent study by Lamp et al. (2015), where the effects of heat stress were separated from the effects of reduced DMI using a pair-feeding model. Specifically, late gestation cows under heat stress retain the ability to effectively mobilize adipose stores similar to the response of pairfed normothermic cows (Lamp et al., 2015). Late gestation heat-stressed cows also mobilize protein reserves, all in an effort to support the demand of the developing fetus. Therefore, calves from heat-stressed dams are challenged before birth and must make physiological accommodation in response to higher heat loads, less effective placental support, and reduced maternal nutrient intake.

The mechanism of heat-stress-induced reductions in gestation length has not been determined, but may be related to altered uterine prostaglandin synthesis and subsequent luteolysis. Late gestation heat stress increased postpartum $\mathrm{PGF}_{2 \alpha}$ concentrations relative to cows that received heat stress abatement, although prepartum PGF $_{2 \alpha}$ was not assessed (Lewis et al., 1984). Early pregnancy heat stress increases $\mathrm{PGF}_{2 \alpha}$ synthesis in cultured uterine endometrium, an effect that may contribute to heat-stress-associated early embryonic losses in vivo (Putney et al., 1988). Because of the increases in circulating cortisol in the fetus and dam 
around parturition, it is reasonable to speculate that heat stress may alter the cortisol response and hasten parturition (Kindahl et al., 2004). However, effects of heat stress on circulating cortisol are inconsistent in cows with increases (Elvinger et al., 1992), decreases (Correa-Calderon et al., 2004), and no change (Dikmen et al., 2008) observed in different studies. Altered $\mathrm{PGF}_{2 \alpha}$ synthesis, therefore, is a likely candidate to drive earlier parturition with heat stress.

A subsequent study in our laboratory indicated that the birth weight deficit observed in calves whose dams are heat stressed do not recover that lost weight even at $1 \mathrm{yr}$ of age, and calves heat stressed in utero are also shorter (Monteiro et al., 2013). A summary of the BW of heat stressed in utero $(\mathbf{H T})$ and cooled $(\mathbf{C L})$ heifer calves at birth, weaning, puberty (i.e., $\sim 12$ mo of age), and calving is presented in Figure 1. It is of interest that the calves born to heat-stressed dams appear to have a postpubertal rebound in weight gain, as we did not observe a difference in BW immediately before or after their first calving relative to the heifers that had developed under cooled conditions in late gestation (Monteiro et al., 2013). Therefore, a low BW observed at birth appears to result in a smaller mature frame size, rather than a lower mature BW.

In addition to weight differences, calves that experience heat stress in utero have altered energy metabolism versus cooled calves. Using insulin and glucose challenges (Tao et al., 2014), we observed that heatstressed calves shunt energy toward peripheral tissues in a manner consistent with greater fat accumulation (Tao and Dahl, 2013). Evidence of adiposity and altered insulin signaling are not available in heat-stressed bovine fetuses, but ovine fetal nutrient restriction results in fatter lambs (De Blasio et al., 2007). Previous work indicates that intrauterine growth restriction (i.e., IUGR) induced by hyperthermia or nutrient restriction results in similar postnatal defects in metabolism characterized by insulin resistance and greater adipose accumulation (Wallace et al., 2005). We have also noted an increase in circulating cortisol in calves from cooled dams relative to those from heat-stressed cows during the preweaning period (Monteiro et al., 2014), further indicating that in utero heat stress alters energy metabolism during the postnatal period. Reduced circulating cortisol in HT calves is consistent with early studies in lambs that reported smaller adrenal cortices in utero heat stressed versus cooled lambs (Ryle and Morris, 1961). Collectively, these changes in energy metabolism are consistent with heat-stressed calves developing smaller frames and greater fat deposition compared with calves from cooled dams.

We recently examined the effect of in utero heat stress on the animal's ability to respond to acute thermal

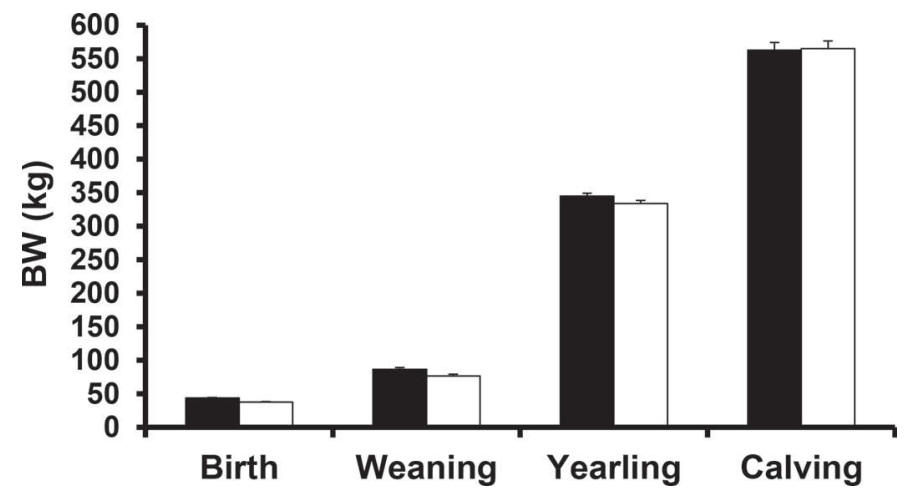

Figure 1. Body weight of heifers that experienced late gestation in utero heat stress (HT; $\mathrm{n}=34$; open bars) or were cooled (CL; $\mathrm{n}=38$; solid bars) through the initial 2 yr of life. Differences were significant between HT and CL at birth, weaning, and through the first $12 \mathrm{mo}$ of age (i.e., birth, weaning, and yearling; $P<0.05$ ), whereas the BW at calving did not differ. Data from Monteiro et al., 2013. Data are presented as LSM \pm SEM.

challenge at maturity (Ahmed et al., 2015). Lactating cows that were either heat stressed in utero $(\mathrm{HT} ; \mathrm{n}=8)$ or cooled ( $\mathrm{CL} ; \mathrm{n}=8$ ) were subjected to ambient temperatures of $\sim 24^{\circ} \mathrm{C}$ without cooling over a 2 -d period. Measurements of rectal temperature, respiration rate, sweating rate, and skin temperature were collected during the challenge. Compared with CL cows, HT cows had lower rectal temperature (CL: $39.18 \pm 0.06$; HT: $38.99 \pm 0.06^{\circ} \mathrm{C} ; P=0.02$ ) and respiration rate (CL: $68.1 \pm 1.6$; HT: $62.2 \pm 1.6 ; P=0.01)$ across the study. Sweating rate tended to be elevated in the CL cows relative to the HT cows (CL: $30.7 \pm 1.6$; HT: $27.1 \pm 1.6$ $\mathrm{g} / \mathrm{m}^{2} \mathrm{~h} ; P=0.12$ ), but skin temperature did not differ between groups. These data support the concept that body growth and metabolic differences in response to in utero heat stress extend to heat tolerance, and suggest that in utero accommodation to heat stress may confer improved ability to respond to heat load at maturity.

\section{HEAT STRESS EFFECTS ON CALF IMMUNE STATUS}

Beyond the effects on growth, in utero heat stress alters immune status of calves relative to those that develop under cooled conditions. We initially observed a reduction in circulating plasma proteins in HT calves compared with CL calves for the first $28 \mathrm{~d}$ of life, even though all calves were managed identically after birth (Tao et al., 2012). Further examination revealed significant reductions of serum concentrations of IgG in calves from heat-stressed dams relative to those from cooled dams $(1,058 \pm 173$ vs. $1,577 \pm 149 \mathrm{mg} / \mathrm{dL}$, respectively; $P=0.03$; Tao et al., 2012). We also observed lower rates of PBMC proliferation in calves following in utero heat stress, and the depressed response persisted 
for at least $56 \mathrm{~d}$ of life (Tao et al., 2012). These observations indicate that late gestation heat stress affects both passive and developing immune competence in the calf relative to those calves that develop under thermoneutral conditions.

Of interest, the effect of in utero heat stress on IgG in the neonate was characterized by reduced apparent efficiency of absorption (AEA) of IgG, indicating that either colostrum or the calf's ability to absorb Ig from that colostrum was negatively affected by in utero heat stress (Tao et al., 2012). With regard to absolute differences in concentrations of $\mathrm{IgG}$ in colostrum, we observed no effect of late gestation heat stress on IgG concentrations, as heat-stressed dry cows have numerically higher concentrations of IgG relative to cooled dams (Tao et al., 2012). Thus, in utero heat stress appears to alter the calf's capacity for Ig transfer.

To more fully understand the mechanism of this difference in AEA, we conducted 2 experiments using calves and colostrum generated from dams that had experienced heat stress or cooling during late gestation (Monteiro et al., 2014). In the first experiment, the HT or CL calves were fed from the same pool of colostrum collected from cows that were not subjected to heat stress when dry. In confirmation of the earlier study of Tao et al. (2012), CL calves had greater AEA compared with HT calves, and growth differences were also observed with CL calves having greater BW at birth and weaning relative to HT calves. For the second experiment, we pooled the colostrum produced by HT or CL cows and fed that to calves born to dams managed under thermoneutral conditions when dry. No differences were found between the groups with regard to AEA or any growth measurements. Subtle effects were found, however, on acquired immune responses during early life that may be related to some factors in the colostrum of CL versus HT dams, yet even those effects did not alter growth or performance trajectories between the groups. Given the importance of passive transfer to calf health and survival, approaches to increase the AEA should be of interest to producers, and heat stress abatement of the dam is an effective method to improve IgG uptake.

\section{EFFECTS OF IN UTERO HEAT STRESS ON PRODUCTIVITY}

The aforementioned effects on calf health and growth are ultimately associated with improved performance. We have analyzed the records of 72 heifers born to heatstressed or cooled dams during the past 5 yr on our university dairy to assess the effect of in utero heat stress on health, reproduction, and milk production (Mon- teiro et al., 2013). It is important to emphasize that following birth all of the calves are managed identically in our system, often within a group, so any observed effects are due to differences induced in utero rather than after birth. Also, the data were collected over a 5 -yr period, limiting the possibility that responses were not due to the only difference in the management of the animals, namely the presence or absence of in utero heat stress. Relative to calves cooled in utero, heifers born to heat-stressed dams left the herd at higher frequency before puberty, usually due to disease. That in utero heat stress led to increased morbidity is not surprising considering the early life immune challenges described above.

Reproductively, heifers that experienced late gestation heat stress were at a disadvantage from conception through lactation compared with herd mates that developed under thermoneutral conditions. Specifically, HT heifers required more services to conceive $(2.6 \pm 0.3$ vs. $1.8 \pm 0.3$, HT vs. CL; $P=0.03$ ), and produced less milk in the first lactation than CL heifers. Differences in age when pregnancy was confirmed were not due to delays in breeding, as the age at first AI did not differ between groups. Further, age at calving did not differ between the HT and CL heifers. The milk yield depression was striking at an average of $5.1 \mathrm{~kg} / \mathrm{d}$ for the first $35 \mathrm{wk}$ of the first lactation (Figure 2). The decrease in milk yield was not associated with lower BW in HT heifers as they were not different from the CL heifers at calving (Figure 1). Seasonal and other management factors were also not different between the groups as all heifers calved across a 5-yr period, and were managed according to normal, industry standard protocols as a group with other young stock on the farm. Thus, it appears that the dam is affected acutely by late gestation heat stress, whereas the calf developing in utero becomes programmed to be less productive for life.

It is likely that adaptations the calf makes to accommodate to the challenging uterine environment under heat stress have lasting physiological effects after birth. Indeed, the calf that is heat stressed in utero exhibits phenotypic changes that are consistent with epigenetic effects (Reynolds et al., 2010; Singh et al., 2012). However, the most studied examples of programming are typically associated with one tissue or disease; thus, the more global effect on lactation in the heat stressed calf is somewhat unique. Although the mammary gland may be altered directly by in utero heat stress, the data collected thus far indicate a coordinated reduction in the efficiency of production rather than a mammaryspecific action (Singh et al., 2012). Certainly future studies are needed to address the nature of the response at the cellular and genomic levels. 


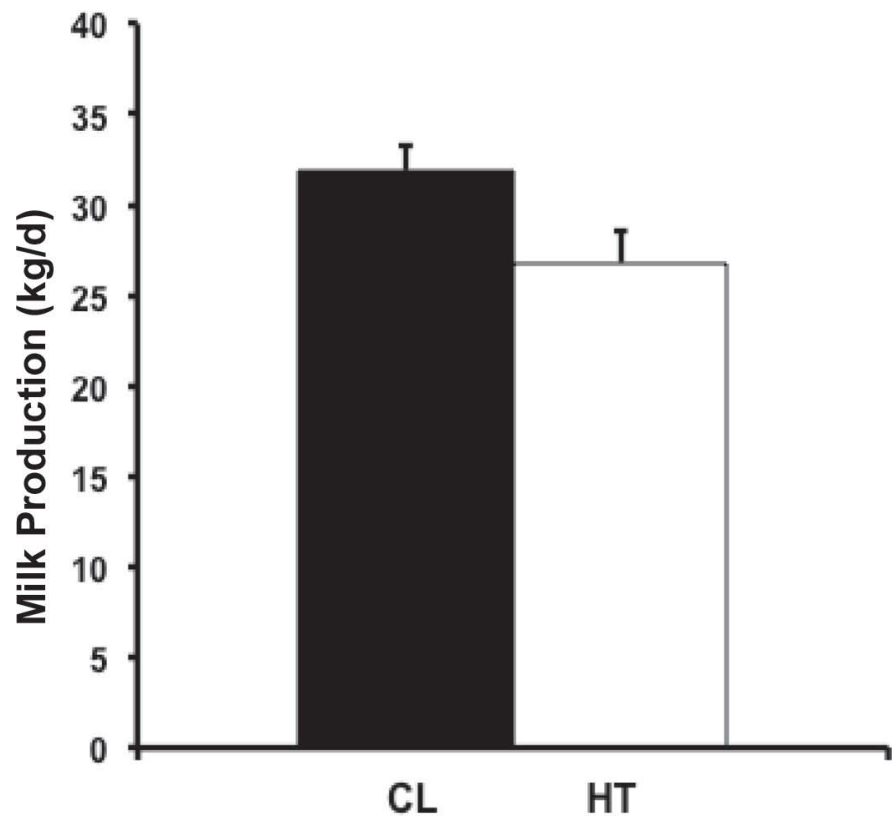

Figure 2. Average daily milk yield of heifers that experienced late gestation in utero heat stress (HT; $\mathrm{n}=29$; open bar) or were cooled ( $\mathrm{CL} ; \mathrm{n}=35$; solid bar), summarized across the initial 35 wk of lactation. Differences were significant between HT and CL $(P<0.05)$. Data from Monteiro et al., 2013. Data are presented as LSM \pm SEM.

\section{CONCLUSIONS}

Substantial evidence is now available to support actively cooling dry cows for the entire dry period (reviewed by Tao and Dahl, 2013). Relative to heat stress, cooling during late gestation increases mammary growth, maintains dry matter intake, and improves immune status during the transition into lactation. However, the most significant benefits of dry cow cooling may be those that accrue to the calves born to cooled dams relative to those that experience heat stress in late gestation. Calves from cooled dams are larger, have a more robust immune system, and have improved reproductive performance compared with those calves born to heat stressed dams. Heifers from cooled dams produce more milk in their first lactation compared with herdmates born to heat stressed dams. More complete understanding of the mechanisms of in utero heat stress on mature production should lead to improvements in production efficiency.

\section{ACKNOWLEDGMENTS}

Many of the studies cited in this review were supported by USDA-Agriculture and Food Research Initiative (AFRI) Integrated Solutions for Animal Agriculture (Washington, DC) and USDA-T-STAR programs
(Washington, DC; awards \#2010-85122-20623 and \#2010-34135-21054 to G. E. Dahl) and the National Science Foundation (Arlington, VA; award \#1247362 to G. E. Dahl), and that support is gratefully acknowledged.

\section{REFERENCES}

Ahmed, B. M., U. Younas, T. Asar, S. Dikman, P. J. Hansen, and G. E. Dahl. 2015. Cows exposed to heat stress in utero exhibit improved thermal tolerance. J. Dairy Sci. 98(Suppl. 2):862. (Abstr.)

Baumgard, L. H., and R. P. Rhoads Jr.. 2013. Effects of heat stress on postabsorptive metabolism and energetics. Annu. Rev. Anim. Biosci. 1:311-337.

Bell, A. W., B. W. McBride, R. Slepetis, R. J. Early, and W. B. Currie. 1989. Chronic heat stress and prenatal development in sheep: I. Conceptus growth and maternal plasma hormones and metabolites. J. Anim. Sci. 67:3289-3299.

Broucek, J., P. Kisac, and M. Uhrincat. 2009. Effect of hot temperatures on the hematological parameters, health and performance of calves. Int. J. Biometeorol. 53:201-208.

Collier, R. J., G. E. Dahl, and M. J. Van Baale. 2006. Major advances associated with environmental effects on dairy cattle. J. Dairy Sci. $89: 1244-1253$.

Collier, R. J., S. G. Doelger, H. H. Head, W. W. Thatcher, and C. J. Wilcox. 1982. Effects of heat stress during pregnancy on maternal hormone concentrations, calf birth weight and postpartum milk yield of Holstein cows. J. Anim. Sci. 54:309-319.

Correa-Calderon, A., D. Armstrong, D. Ray, S. DeNise, M. Enns, and C. Howison. 2004. Thermoregulatory responses of Holstein and Brown Swiss heat-stressed dairy cows to two different cooling systems. Int. J. Biometeorol. 48:142-148.

De Blasio, M. J., K. L. Gatford, I. C. McMillen, J. S. Robinson, and J. A. Owens. 2007. Placental restriction of fetal growth increases insulin action, growth, and adiposity in the young lamb. Endocrinology 148:1350-1358.

Dikmen, S., E. Alava, E. Pontes, J. M. Fear, B. Y. Dikmen, T. A. Olson, and P. J. Hansen. 2008. Differences in thermoregulatory ability between slick-haired and wild-type lactating Holstein cows in response to acute heat stress. J. Dairy Sci. 91:3395-3402.

Elvinger, F., R. P. Natzke, and P. J. Hansen. 1992. Interactions of heat stress and bovine somatotropin affecting physiology and immunology of lactating cows. J. Dairy Sci. 75:449-462.

Kelley, D. W., C. A. Osborne, J. F. Evermann, S. M. Parish, and C. T. Gaskins. 1982. Effects of chronic heat and cold stressors on plasma immunoglobulin and mitogen-induced blastogenesis in calves. J. Dairy Sci. 65:1514-1528.

Kindahl, H., B. Kornmatitsuk, and H. Gustafsson. 2004. The cow in endocrine focus before and after calving. Reprod. Domest. Anim. $39: 217-221$.

Lacetera, N., U. Bernabucci, D. Scalia, B. Ronchi, G. Kuzminsky, and A. Nardone. 2005. Lymphocyte functions in dairy cows in hot environment. Int. J. Biometeorol. 50:105-110.

Lamp, O., M. Derno, W. Otten, M. Mielenz, G. Nürnberg, and B. Kuhla. 2015. Metabolic heat stress adaption in transition cows: Differences in macronutrient oxidation between late-gestating and early-lactating German Holstein dairy cows. PLoS ONE 10:e0125264

Lewis, G. S., W. W. Thatcher, E. L. Bliss, M. Drost, and R. J. Collier. 1984. Effects of heat stress during pregnancy on postpartum reproductive changes in Holstein cows. J. Anim. Sci. 58:174-186.

Monteiro, A. P. A., S. Tao, I. M. Thompson, and G. E. Dahl. 2013. Effect of heat stress in utero on calf performance and health through the first lactation. J. Anim. Sci. 91(Suppl. 1):184. (Abstr.)

Monteiro, A. P. A., S. Tao, I. M. Thompson, and G. E. Dahl. 2014. Effect of heat stress during late gestation on immune function and growth performance of calves: Isolation of altered colostral and calf factors. J. Dairy Sci. 97:6426-6439. 
O'Brien, M. D., R. P. Rhoads, S. R. Sanders, G. C. Duff, and L. H Baumgard. 2010. Metabolic adaptations to heat stress in growing cattle. Domest. Anim. Endocrinol. 38:86-94.

Putney, D. J., J. R. Malayer, T. S. Gross, W. W. Thatcher, P. J. Hansen, and M. Drost. 1988. Heat stress-induced alterations in the synthesis and secretion of proteins and prostaglandins by cultured bovine conceptuses and uterine endometrium. Biol. Reprod. 39:717-728.

Reynolds, L. P., P. P. Borowicz, J. S. Caton, K. A. Vonnahme, J. S. Luther, C. J. Hammer, K. R. Maddock Carlin, A. T. Grazul-Bilska, and D. A. Redmer. 2010. Developmental programming: The concept, large animal models, and the key role of uteroplacental vascular development. J. Anim. Sci. 88:E61-E72.

Rhoads, M. L., J. W. Kim, R. J. Collier, B. A. Crooker, Y. R. Boisclair, L. H. Baumgard, and R. P. Rhoads. 2010. Effects of heat stress and nutrition on lactating Holstein cows: II. Aspects of hepatic growth hormone responsiveness. J. Dairy Sci. 93:170-179.

Ryle, M., and L. R. Morris. 1961. Some quantitative studies on tissues of lambs dwarfed by high temperatures during gestation. Aust. J. Exp. Biol. Med. Sci. 39:79-91.

Singh, K., A. J. Molenaar, K. M. Swanson, B. Gudex, J. A. Arias, R. A. Erdman, and K. Stelwagen. 2012. Epigenetics: A possible role in acute and transgenerational regulation of dairy cow milk production. Animal 6:375-381.

Tao, S., and G. E. Dahl. 2013. Invited review: Heat stress impacts during late gestation on dry cows and their calves. J. Dairy Sci. 96:4079-4093.

Tao, S., A. P. Monteiro, I. M. Thompson, M. J. Hayen, and G. E. Dahl. 2012. Effect of late gestation maternal heat stress on growth and immune function of dairy calves. J. Dairy Sci. 95:7128-7136.

Tao, S., A. P. A. Monteiro, M. J. Hayen, and G. E. Dahl. 2014. Short Communication: Maternal heat stress during the dry period alters whole body insulin response of calves postnatally. J. Dairy Sci. 97:897-901.

Thompson, I. M., S. Tao, J. Branen, A. D. Ealy, and G. E. Dahl. 2013. Environmental regulation of pregnancy-specific protein B concentrations during late pregnancy in dairy cattle. J. Anim. Sci. 91:168-173.

Wallace, J. M., T. R. Regnault, S. W. Limesand, W. W. Hay Jr., and R. V. Anthony. 2005. Investigating the causes of low birth weight in contrasting ovine paradigms. J. Physiol. 565:19-26. 